\title{
OTIMIZAÇÃO DE PROTOCOLO DE PREPARAÇÃO DE AMOSTRAS EM LABORATÓRIO DE ANÁLISES FÍSICAS*
}

\author{
Flávia Guimarães Rocha Godinho Campos Vargas ${ }^{1}$ \\ Warlei Gonçalves Xavier Rodrigues ${ }^{2}$ \\ Ana Carolina Chieregati ${ }^{3}$ \\ Éder Lúcio Martins ${ }^{4}$
}

\section{Resumo}

Amostragem pode ser definida como a sequência de operações que visam retirar do todo (lote) uma parcela (amostra) que conserve as propriedades de interesse do lote. O menor erro possível em um protocolo de amostragem é chamado de erro fundamental de amostragem (FSE), o qual está associado à heterogeneidade constitucional do minério e pode ser estimado através de testes de heterogeneidade. A realização do teste de heterogeneidade com minério fonte de zinco, proveniente da mina de Vazante, permitiu concluir que a heterogeneidade da willemita é menor que a heterogeneidade de minérios de ouro e maior que a de minérios fonte de alumínio, por exemplo. Além disso, os resultados do teste de heterogeneidade permitiram a otimização do protocolo de preparação de amostras do laboratório físico, reduzindo o erro total de 13,4\% para 6,0\% sem investimentos em infraestrutura de laboratório.

Palavras-chave: Amostragem; Erro fundamental; Willemita; Teste de heterogeneidade.

\section{OPTIMIZATION OF SAMPLES PREPARATION PROTOCOL IN LABORATORY OF PHYSICAL ANALISIS}

\section{Abstract}

Sampling can be define as a sequence of operations which aim to obtain a fraction (sample) that represents the lot and keep unaltered the characteristics of interest to be analyzed. The minor possible error of a sampling protocol is the fundamental sampling error (FSE) associated to the ore constitutional heterogeneity and calculate through the heterogeneity test. The execution of such test on zinc ore from Vazante mine concluded that willemite heterogeneity is lower than gold ores one and greater than alumina ores, for example. Moreover, the results guided the optimization of samples preparation protocol in laboratory of physical analysis by reducing the total error from $13.4 \%$ to $6.0 \%$ without any investment on infrastructure.

Keywords: Sampling; Fundamental sampling error; Willemita; Heterogeneity Test.

1 Engenheiro de Minas, Graduado, Engenheiro de Processo Pleno, Processo, Votorantim Metais, Brasil.

2 Engenheiro de Minas, Graduado, Técnico, Processo, Votorantim Metais, Brasil.

3 Engenheira de Minas, Professora, Departamento de Engenharia de Minas e de Petróleo, Escola Politécnica da USP, São Paulo, Brasil.

4 Engenheiro Metalurgista, Mestre, Gerente de Tecnologia, Tecnologia, Votorantim Metais. 


\section{INTRODUÇÃO}

Amostragem pode ser definida como uma sequência de operações que visa retirar do todo (lote) uma parcela (amostra) que conserve inalteradas as características de interesse. $\mathrm{Na}$ indústria mineral a amostragem é praticada desde a avaliação da viabilidade do empreendimento até a entrega do produto final ao cliente. Portanto, é parte de todas as etapas do processo produtivo e tem potencial de influenciar a tomada de decisões diária.

Quando realizados de forma correta, os trabalhos de amostragem contribuem para otimizar processos e alavancar resultados. Em contrapartida, as decisões guiadas por resultados decorrentes de uma amostragem incorreta podem desperdiçar tempo e recursos da empresa e, em casos extremos, inviabilizar o empreendimento mineiro. A tomada de amostras não representativas é consequência da adição de erros durante as diversas etapas da operação de amostragem. Segundo Pitard (2009, apud Chieregati \& Pitard, 2011), o erro total de amostragem (TSE) é a soma dos seguintes erros:

- Erro fundamental de amostragem (FSE)

- Erro de segregação e grupamento (GSE)

- Erro de flutuação da heterogeneidade (HFE)

- Erro de flutuação de qualidade (QFE)

- Erro de ponderação do incremento (IWE)

- Erro de materialização do incremento (IME)

- Erro de delimitação do incremento (IDE)

- Erro de extração do incremento (IEE)

- Erro de preparação do incremento (IPE)

Ou seja, quando todos os erros citados ocorrem individualmente, o erro total da amostragem (TSE) pode ser definido pela Equação 1 abaixo:

$$
\mathrm{TSE}=\mathrm{FSE}+\mathrm{GSE}+\mathrm{HFE}+\mathrm{QFE}+\mathrm{IWE}+\mathrm{IME}+\mathrm{IDE}+\mathrm{IEE}+\mathrm{IPE}
$$

$\mathrm{O}$ erro fundamental de amostragem (FSE) é consequência da heterogeneidade constitucional $\left(\mathrm{CH}_{L}\right)$ de um determinado lote. Portanto, este é o menor erro existente em um protocolo, pois mesmo em condições ideais de amostragem, quando todos os demais erros são nulos, há a heterogeneidade própria do lote e o erro fundamental de amostragem (FSE) persiste.

Devido à dificuldade de calcular a heterogeneidade constitucional do lote $\left(\mathrm{CH}_{L}\right)$, na prática, calcula-se o fator constante de heterogeneidade constitucional $\left(\mathrm{IH}_{L}\right)$ pela multiplicação de outros fatores definidos por Pierre Gy (1998), conforme Equação 2 abaixo:

$$
\left(\mathrm{IH}_{L}\right)=\mathrm{c} \text { I } \mathrm{g} \mathrm{d}^{3}
$$

Onde $d$ é tamanho máximo de partículas, $c$ é o fator mineralogia, lé o fator liberação, $f$ é o fator forma e $g$ é o fator granulometria. Os fatores $c, l, f$ e $g$ podem ser estimados experimentalmente, no entanto, é uma determinação difícil. A estimativa experimental do fator de heterogeneidade constitucional, por meio de testes de heterogeneidade, é mais recomendada que o uso dos fatores da Equação 2, visto que permite calibrar o $\left(\mathrm{IH}_{L}\right)$ para cada tipo específico de minério, gerando resultados mais confiáveis. $\mathrm{O}$ conhecimento da heterogeneidade do minério é de extrema importância para a otimização de protocolos de amostragem e preparação de amostras, de forma a caracterizar a heterogeneidade intrínseca do minério e definir as massas mínimas representativas em cada etapa do processo, minimizando os erros decorrentes da amostragem. 


\section{MATERIAIS E MÉTODOS}

Para a realização do teste de heterogeneidade com minério willemita foi coletada uma amostra de aproximadamente $300 \mathrm{~kg}$ na britagem $\mathrm{W}$ da usina de concentração de Vazante. Todo o material foi submetido a peneiramento em cinco malhas de aberturas $25,4 \mathrm{~mm}, 12,7 \mathrm{~mm}, 6,35 \mathrm{~mm}, 4,75 \mathrm{~mm}$ e $3,35 \mathrm{~mm}$, formando quatro frações granulométricas distintas: "- $25,4 \mathrm{~mm}+12,7 \mathrm{~mm}$ ", "- $12,7 \mathrm{~mm}+6,35 \mathrm{~mm}$ ", "-6,35mm $+4,75 \mathrm{~mm}$ " e "-4,75mm $+3,35 \mathrm{~mm}$ ".

Cada uma das quatro frações granulométricas foi então homogeneizada e disposta sobre uma matriz $5 \times 10$ desenhada em uma bancada de tamanho adequado, de forma que foi possível realizar a coleta de fragmentos individuais, de forma aleatória, com chances iguais de coleta para todos os fragmentos. Na Figura 1 a seguir, podese observar a disposição dos fragmentos na malha da fração $-25,4 \mathrm{~mm}+12,7 \mathrm{~mm}$.

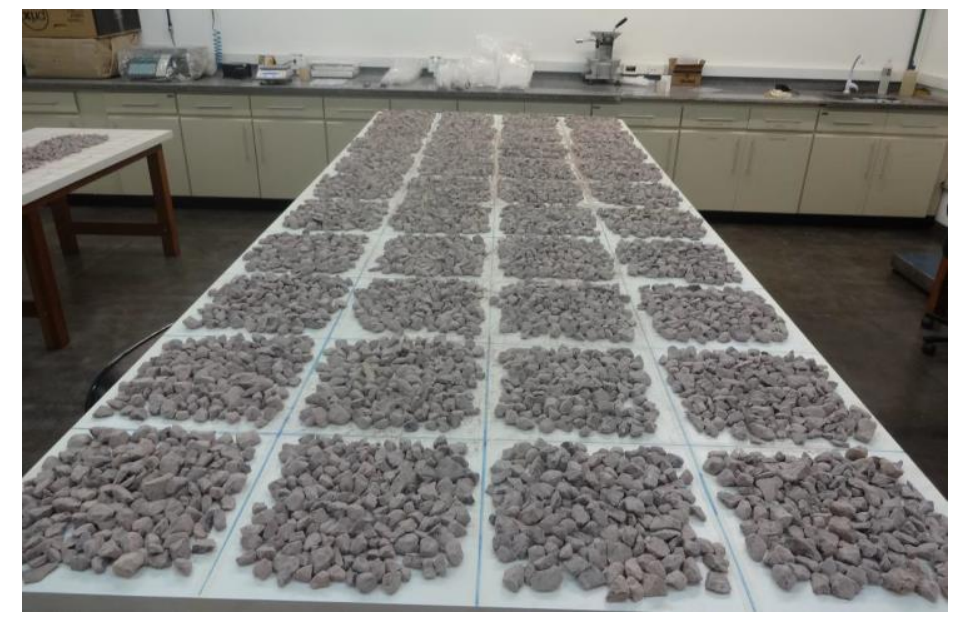

Figura 1. Fragmentos $-25,4 \mathrm{~mm}+12,7 \mathrm{~mm}$ dispostos na malha de teste de heterogeneidade

Após a disposição da amostra na matriz $5 \times 10$, foi realizada a coleta aleatória de um fragmento por quadro, totalizando 50 fragmentos por amostra. Este método de seleção de amostra elimina todos os erros de amostragem, exceto o erro fundamental de amostragem (FSE), o qual caracteriza a heterogeneidade constitucional do minério. No total foram coletadas 50 amostras compostas por 50 fragmentos de cada uma das quatro frações granulométricas, totalizando 200 amostras, colocadas em sacos plásticos previamente identificados.

As análises químicas para obtenção dos teores de zinco (\%) foram realizadas via complexometria.

Com os resultados de massa e análise química de cada um dos 50 grupos de amostras pode-se, para cada uma das frações granulométricas analisadas, estimar o valor do fator de heterogeneidade constitucional EST $\left(\mathrm{IH}_{L}\right)$ através da Equação 3 abaixo:

$$
\text { EST IHL }=g \sum \frac{\left(a_{q}-a_{Q}\right)^{2}}{a_{Q}{ }^{2}} \frac{M_{q}{ }^{2}}{M_{Q}}
$$

Onde $\mathrm{M}_{\mathrm{q}}$ e $\mathrm{a}_{\mathrm{q}}$ são respectivamente a massa e teor de cada uma das 50 amostras geradas em cada uma das frações; $\mathrm{MQ}_{\mathrm{Q}}$ e aQ são o somatório de acordo com a Equação 4 e a Equação 5, respectivamente:

$$
\begin{aligned}
& M_{Q}=\Sigma M_{q} \\
& a_{Q}=\frac{1}{M_{Q}} \sum a_{Q} M_{q}
\end{aligned}
$$


O diâmetro nominal para cada uma das frações granulométricas pode ser obtido através da Equação 6 abaixo:

$$
d=\sqrt[3]{\frac{d_{1}^{3}+d_{2}^{3}}{2}}
$$

\section{RESULTADOS E DISCUSSÃO}

A Tabela 1 abaixo apresenta os resultados de massa e teor de zinco (\%) para as amostras geradas pelo teste de heterogeneidade.

Tabela 1.Identificação, massas e teores de $\mathrm{Zn}$ das amostras referentes ao teste de heterogeneidade

\begin{tabular}{|c|c|c|c|c|c|c|c|c|c|c|c|c|}
\hline \multirow{2}{*}{$\begin{array}{l}\text { size fraction } \\
\text { \# sample }\end{array}$} & \multicolumn{3}{|c|}{$-4,75+3,35 \mathrm{~mm}$} & \multicolumn{3}{|c|}{$-6,35+4,75 \mathrm{~mm}$} & \multicolumn{3}{|c|}{$-12,7+6,35 \mathrm{~mm}$} & \multicolumn{3}{|c|}{$-25,4+12,7 \mathrm{~mm}$} \\
\hline & ID & mass $(g)$ & $\mathrm{Zn}(\%)$ & ID & mass (g) & $\mathrm{Zn}(\%)$ & ID & mass (g) & $\mathrm{Zn}(\%)$ & ID & mass (g) & $\mathrm{Zn}(\%)$ \\
\hline 1 & $\mathrm{TH} 301$ & 59,00 & 6,57 & TH 401 & 133,10 & 9,34 & TH 601 & 243,90 & 6,86 & TH 1201 & 842,60 & 10,95 \\
\hline 2 & TH 302 & 56,60 & 7,13 & TH 402 & 126,70 & 11,27 & TH 602 & 225,20 & 8,29 & TH 1202 & 892,20 & 4,84 \\
\hline 3 & TH 303 & 60,40 & 7,54 & TH 403 & 138,40 & 9,04 & TH 603 & 179,00 & 7,01 & TH 1203 & 840,80 & 8,85 \\
\hline 4 & TH 304 & 61,90 & 7,14 & TH 404 & 134,50 & 8,37 & TH 604 & 161,10 & 4,59 & TH 1204 & 979,90 & 8,08 \\
\hline 5 & TH 305 & 64,40 & 5,77 & TH 405 & 123,40 & 10,66 & TH 605 & 154,70 & 4,69 & TH 1205 & 824,10 & 8,61 \\
\hline 6 & TH 306 & 59,10 & 5,65 & TH 406 & 120,80 & 10,59 & TH 606 & 250,80 & 9,25 & TH 1206 & 876,00 & 7,48 \\
\hline 7 & TH 307 & 61,30 & 7,37 & TH 407 & 119,50 & 9,38 & TH 607 & 245,00 & 3,26 & TH 1207 & 812,70 & 6,87 \\
\hline 8 & TH 308 & 58,70 & 6,15 & TH 408 & 127,40 & 9,42 & TH 608 & & 5,02 & TH 1208 & 833,80 & 3,45 \\
\hline 9 & TH 309 & 62,70 & 6,61 & TH 409 & 119 & 9,88 & TH 609 & & 3,32 & TH 1209 & & 10,39 \\
\hline 10 & TH 310 & 62,20 & 7,06 & TH 410 & 128,30 & 7,47 & TH 610 & & 4,84 & TH 1210 & & 9,24 \\
\hline 11 & TH 311 & 61,60 & 8,52 & TH 411 & 130,10 & 7,46 & TH 611 & 253,40 & 3,76 & TH 1211 & 823,50 & 9,99 \\
\hline 12 & TH 312 & 59,20 & 6,23 & TH 412 & 122,20 & 8,02 & TH 612 & 218,40 & 6,61 & TH 1212 & 945,20 & 7,82 \\
\hline 13 & TH 313 & 60,50 & 8,38 & $\mathrm{TH} 413$ & & 9,52 & TH 613 & & 6,41 & TH 1213 & & 6,01 \\
\hline 14 & TH 314 & 63,00 & 5,94 & TH 414 & 128,10 & 9,37 & TH 614 & & 5,48 & TH 1214 & & 11,47 \\
\hline 15 & TH 315 & 60,50 & 8,42 & TH 415 & & 11,73 & TH 615 & & 11,31 & TH 1215 & & 11,60 \\
\hline 16 & TH 316 & 61,00 & 8,11 & TH 416 & 127,80 & 11,72 & TH 616 & 221,60 & 9,11 & $\mathrm{TH} 1216$ & 821,60 & 9,13 \\
\hline 17 & TH 317 & 58,70 & 6,60 & TH 417 & & 8,98 & TH 617 & & 8,22 & TH 1217 & & 99 \\
\hline 18 & TH 318 & 61,7 & 6,41 & TH 418 & & 7,61 & TH 618 & & 7,01 & TH 1218 & & 6,15 \\
\hline 19 & TH 319 & 63, & 7,99 & TH 419 & & 8,61 & TH 619 & & 8,94 & TH 1219 & & 6,67 \\
\hline 20 & TH 320 & 62,00 & 7,76 & TH 420 & 123,00 & 8,96 & TH 620 & 163,20 & 7,73 & TH 1220 & 712,50 & 10,40 \\
\hline 21 & TH 321 & 55,40 & 7,55 & TH 421 & 117,60 & 9,78 & TH 621 & & 6,47 & TH 1221 & 778,40 & 9,73 \\
\hline 22 & TH 322 & 58, & 6,83 & TH 422 & & 30 & TH 622 & & 6,66 & TH 1222 & & 10,50 \\
\hline 23 & TH 323 & 58,10 & 7,8 & TH 423 & 121,80 & 7,34 & TH 623 & 224 & 7,75 & TH 1223 & 3,30 & 7,34 \\
\hline 24 & TH 324 & 59,20 & 8,66 & TH 424 & 116,20 & 9,03 & TH 624 & 229,70 & 2,42 & TH 1224 & & 9,66 \\
\hline 25 & TH 325 & 58,80 & 7,53 & TH 425 & 118,50 & 10,26 & TH 625 & 223,50 & 3,77 & TH 1225 & 761,50 & 8,98 \\
\hline 26 & TH 336 & 59 , & 7,61 & TH 426 & & 10,49 & TH 626 & & 5,46 & TH 1226 & & 58 \\
\hline 27 & TH 327 & 65,6 & 8,52 & TH 427 & & 6,73 & TH 627 & & 10,94 & TH 1227 & 1,20 & 11,52 \\
\hline 28 & TH 328 & 61,00 & 7,25 & TH 428 & & 9,16 & TH 628 & & 8,39 & TH 1228 & & 9,50 \\
\hline 29 & TH 329 & 59,00 & 7,57 & TH 429 & 116,30 & 7,23 & TH 629 & 178 & 3,89 & TH 1229 & 653,80 & 9,15 \\
\hline 30 & & 60 , & 6,88 & TH 430 & & 7,74 & TH 630 & & 8, & 1230 & & 29 \\
\hline 31 & TH 331 & 59,1 & 8,65 & TH 431 & & 9,01 & TH 631 & & 10,00 & $\mathrm{TH} 1231$ & & 9,91 \\
\hline 32 & TH 332 & 60,40 & 6,59 & TH 432 & & 8,29 & TH 632 & & 7,81 & TH 1232 &, 50 & 12,40 \\
\hline 33 & TH 333 & 58,00 & 6,87 & TH 433 & 118,00 & 8,58 & TH 633 & 198,00 & 5,98 & TH 1233 & 692,70 & 6,99 \\
\hline 34 & TH 334 & 57,50 & 7,99 & TH 434 & & 8,53 & TH 634 & & 7,05 & TH 1234 & 749,40 & 8,64 \\
\hline 35 & & 61 & 7,55 & TH 435 & & 7,89 & TH 635 & & 7,38 & 1235 & & \\
\hline 36 & TH 336 & 60,90 & 8,14 & TH 436 & 121,20 & 7,24 & TH 636 & 201,80 & 9,31 & TH 1236 & 838,70 & 7,14 \\
\hline 37 & TH 337 & 61,10 & 8,02 & TH 437 & 11,20 & 10,20 & TH 637 & 191,10 & 12,82 & TH 1237 & 649,90 & 6,82 \\
\hline 38 & TH 338 & 58,80 & 7,40 & TH 438 & & 8,01 & TH 638 & & 9,90 & TH 1238 & 731,20 & 3,07 \\
\hline 39 & & 60 , & 8,07 & TH 439 & & & TH 639 & & $3, \varepsilon$ & TH 1239 & & 11,44 \\
\hline 40 & $\mathrm{TH} 340$ & 61,2 & 8,35 & $\mathrm{TH} 440$ & & 6,73 & TH 640 & & 7,91 & TH 1240 & 828,00 & 10,05 \\
\hline 41 & TH 341 & 60,50 & 7,22 & TH 441 & 27,60 & 10,25 & TH 641 & 197 & 8,68 & TH 1241 & 733,60 & 7,31 \\
\hline 42 & TH 342 & 58,20 & 7,79 & TH 442 & 119,60 & 10,20 & TH 642 & 206 & 8,48 & TH 1242 & 775,30 & 10,72 \\
\hline 43 & TH 343 & 56,70 & 8,39 & TH 443 & & 10,62 & TH 643 & & 8,15 & TH 1243 & & 14,16 \\
\hline 44 & & 63,3 & & TH 444 & & 7,55 & TH 644 & & 9,1 & 1244 & & \\
\hline 45 & 345 & 57,90 & 5,83 & TH 445 & & 7,52 & TH 645 & 207,40 & 4,64 & TH 1245 & 820,10 & 13,38 \\
\hline 46 & TH 346 & 60,30 & 6,76 & TH 446 & 123,30 & 9,32 & TH 646 & 145,50 & 6,37 & TH 1246 & 834,40 & 11,59 \\
\hline 47 & TH 347 & 60,80 & 9,16 & TH 447 & 123,60 & 9,14 & TH 647 & 198,40 & 4,51 & TH 1247 & 823,00 & 14,85 \\
\hline 48 & & 58,20 & & TH 448 & & 9,97 & TH 648 & & 5,35 & TH 1248 & & 8,92 \\
\hline 49 & 4349 & 58,30 & 5,68 & TH 449 & 118,90 & 9,84 & TH 649 & 185 , & 5,09 & TH 1249 & 737,70 & 9,96 \\
\hline 50 & TH 350 & 58,10 & 7,99 & TH 450 & 121,00 & 8,33 & TH 650 & 165,10 & 7,73 & TH 1250 & 718,90 & 10,79 \\
\hline total mass $\left(\mathrm{M}_{\mathrm{EL}}\right)$ & & 3004,8 & & & 6030,4 & & & 10246,1 & & & 39303,9 & \\
\hline mean & & 60,1 & 7,36 & & 120,6 & 9,00 & & 204,9 & 6,92 & & 786,08 & 9,11 \\
\hline abs std deviation & & & 0,895 & & & 1,258 & & & 2,300 & & & 2,397 \\
\hline I std deviation (\%) & & & 12,2 & & & 14,0 & & & 33,2 & & & 26,3 \\
\hline variance & & & 0,801 & & & 1,583 & & & 5,288 & & & 5,744 \\
\hline
\end{tabular}


A Tabela 2 abaixo apresenta os resultados do cálculo do valor estimado de $\left(\mathrm{IH}_{L}\right)$ conforme Equação 3, bem como dos diâmetros nominais conforme Equação 6.

Tabela 2. Constantes de heterogeneidade para os respectivos diâmetros nominais

\begin{tabular}{cc}
$\begin{array}{c}\text { Diâmetros } \\
\text { Nominais }(\mathbf{c m})\end{array}$ & EST $\left(\mathbf{I H}_{\mathbf{L}}\right)$ \\
\hline 0,42 & 0,48 \\
\hline 0,57 & 1,31 \\
\hline 1,05 & 13,19 \\
\hline 2,10 & 30,41 \\
\hline
\end{tabular}

A partir dos valores apresentados na Tabela 2 é possível construir um gráfico di-log (Figura 2) e determinar os valores de $k$ e $\alpha$.

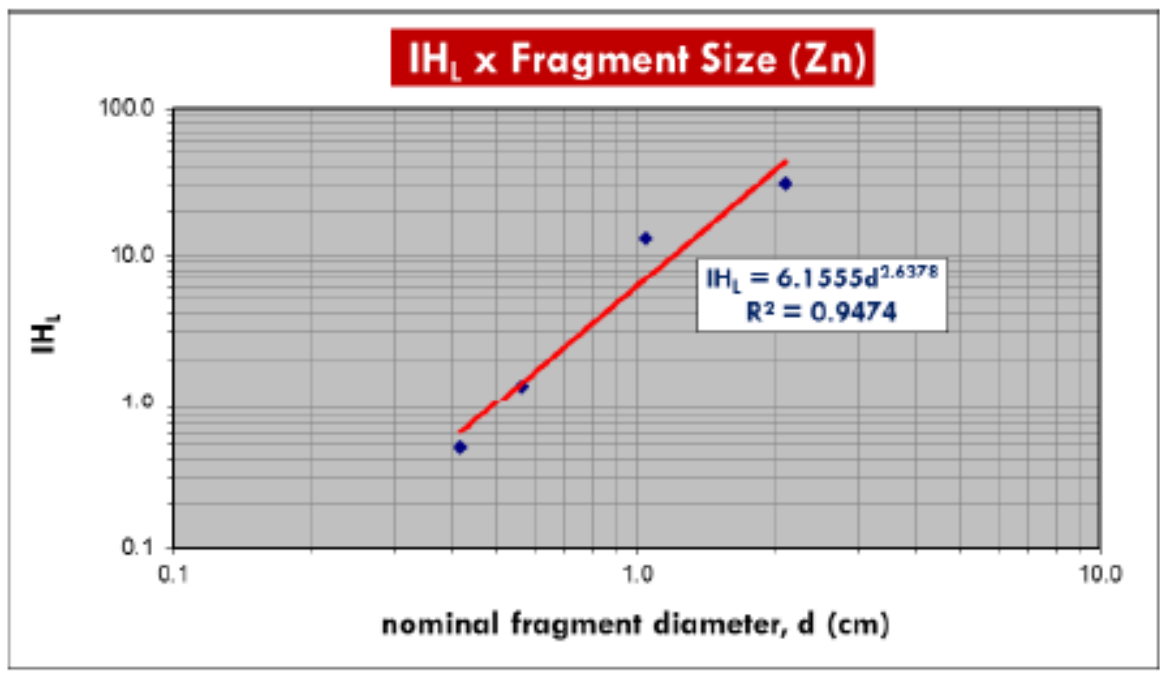

Figura 2. Correlação entre $(\mathrm{IH} \mathrm{L})$ e tamanho do fragmento

Portanto, para o minério Willemita, as constantes calibradas pelo teste de heterogeneidade são $K=6,155$ e $\alpha=2,6378$. Essas constantes indicam a heterogeneidade do minério, ou seja, quanto maiores $K$ e $\alpha$, maior a heterogeneidade do minério. Valores típicos de K para minério de ouro variam de 50 a 1000, enquanto que para bauxita variam de 0,05 a 0,10 . Os valores de $\alpha$ variam, normalmente, de 1 a 3 independentemente do tipo de minério. Portanto, pode-se afirmar que a heterogeneidade do minério de vazante é menor que a heterogeneidade de minérios de ouro e maior que a de minérios de alumínio, por exemplo.

A variância $\mathrm{s}^{2}$ FSE do erro fundamental para este minério pode ser escrita conforme Equação 7 abaixo:

$$
\mathrm{S}^{2} \mathrm{FSE}=6,1555 \mathrm{~d}^{2,6378}\left(\frac{1}{M s}-\frac{1}{M l}\right)
$$

A partir disto, é possível calcular a variância do erro fundamental de amostragem $s^{2} \mathrm{FSE}$ e os desvios SFSE para o atual protocolo de preparação de amostras primárias. A Tabela 3 abaixo apresenta os resultados: 
Tabela 3. Variância do erro fundamental e desvios para o protocolo atual de amostragem

\section{CURRENT PROTOCOL}

\begin{tabular}{lcccccc}
\hline \multicolumn{1}{c}{ stage } & $\begin{array}{c}\text { initial } \\
\text { mass } \mathbf{( g )}\end{array}$ & $\begin{array}{c}\text { final } \\
\text { mass }(\mathbf{g})\end{array}$ & $\begin{array}{c}\mathbf{d}_{\mathbf{N}} \\
(\mathbf{c m})\end{array}$ & $\begin{array}{c}\mathbf{I H}_{\mathbf{L}} \\
(\mathbf{g})\end{array}$ & $\begin{array}{c}\text { rel var } \\
\left(\mathbf{s}^{2}{ }_{\text {FSE }}\right)\end{array}$ & $\begin{array}{c}\text { rel dev } \\
\left(\mathbf{s}_{\text {FSE rel }}\right)\end{array}$ \\
\hline 1. Primary sampling & 16.000 .000 & 5.000 & 0,95 & 5,38 & 0,001075 & $3,28 \%$ \\
\hline 2. Quartering (Jones) & 5.000 & 300 & 0,95 & 5,38 & 0,016846 & $12,98 \%$ \\
\hline 3. Disc grinding & 300 & 300 & 0,03 & 0,00059 & 0,000000 & $0,00 \%$ \\
\hline 4. Quartering (scoop) & 300 & 20 & 0,03 & 0,00059 & 0,000028 & $0,53 \%$ \\
\hline 5. Pulverization & 20 & 20 & 0,0074 & 0,000015 & 0,000000 & $0,00 \%$ \\
\hline 6. Selection of analytical sam & 20 & 20 & 0,0074 & 0,000015 & 0,000000 & $0,00 \%$ \\
\hline \multicolumn{1}{c}{ TOTAL } & & & & & $\mathbf{0 , 0 1 7 9 4 9}$ & $\mathbf{1 3 , 4 0 \%}$ \\
\hline
\end{tabular}

Nota-se que a etapa crítica do processo de preparação atual é o quarteamento da amostra primária, sendo necessário reduzir este erro para $5 \%$, valor máximo recomendado por Pitard (1993). A redução do erro passa obrigatoriamente pelo aumento da massa retirada ou pela redução da granulometria, o que implicaria em mais uma etapa de cominuição no protocolo.

A Tabela 4 apresenta o protocolo otimizado, de modo a garantir um desvio padrão relativo do erro fundamental inferior a $5 \%$ em cada uma das etapas de preparação.

Tabela 4. Variância do erro fundamental e desvios para o protocolo otimizado de amostragem

\begin{tabular}{|c|c|c|c|c|c|c|}
\hline \multicolumn{7}{|c|}{ OPTMIZED PROTOCOL } \\
\hline stage & $\begin{array}{c}\text { initial } \\
\text { mass }(g)\end{array}$ & $\begin{array}{c}\text { final } \\
\text { mass (g) }\end{array}$ & $\begin{array}{c}d_{N} \\
(\mathrm{~cm})\end{array}$ & $\begin{array}{l}\mathrm{IH}_{\mathrm{L}} \\
(\mathrm{g})\end{array}$ & $\begin{array}{l}\text { rel var } \\
\left(\mathrm{s}_{\mathrm{FSE}}^{2}\right)\end{array}$ & $\begin{array}{l}\text { rel dev } \\
\left(S_{\mathrm{FSE}} \text { rel }\right)\end{array}$ \\
\hline 1. Primary sampling & 1.000 .000 .000 & 5.000 & 0,95 & 5,38 & 0,001075 & $3,28 \%$ \\
\hline 2. Quartering (Jones) & 5.000 & 1.500 & 0,95 & 5,38 & 0,002509 & $5,01 \%$ \\
\hline 3. Disc grinding & 1.500 & 1.500 & 0,03 & 0 & 0,000000 & $0,00 \%$ \\
\hline 4. Quartering (scoop) & 1.500 & 20 & 0,03 & 0,00059 & 0,000029 & $0,54 \%$ \\
\hline 5. Pulverization & 20 & 20 & 0,0074 & 0,000015 & 0,000000 & $0,00 \%$ \\
\hline 6. Selection of analytical sam & 20 & 20 & 0,0074 & 0,000015 & 0,000000 & $0,00 \%$ \\
\hline TOTAL & & & & & 0,003614 & $6,01 \%$ \\
\hline
\end{tabular}

Observa-se que a seleção de uma alíquota de 1,5 kg em vez de $300 \mathrm{~g}$ na etapa de quarteamento primário reduz o erro dessa etapa de $12,98 \%$ para $5,01 \%$. Essa proposta de otimização, além de reduzir o erro, é de fácil aplicação prática pois não necessita de investimentos em equipamentos e não adiciona etapas (e, consequentemente, outras fontes de erros) no protocolo.

\section{CONCLUSÃO}

O erro fundamental de amostragem (FSE) é intrínseco ao processo e está relacionado à heterogeneidade constitucional de cada tipo de minério. O teste de heterogeneidade é uma ferramenta que possibilita estimar a variância do erro fundamental $\left(\mathrm{s}^{2} \mathrm{FSE}\right) \mathrm{e}$ trabalhar na otimização de protocolos de amostragem através da sua minimização. $A$ realização do teste de heterogeneidade com o minério de zinco de Vazante e a aplicação de seus resultados no protocolo de preparação do laboratório físico possibilitaram reduzir o erro de preparação de amostras de $13,4 \%$ para 6,01\%, apenas aumentando a massa de amostra resultante do quarteamento primário. 
Consequentemente, a confiabilidade nos resultados de teores das amostras enviadas ao laboratório aumentou significativamente.

\section{Agradecimentos}

À Votorantim Metais - Vazante pela oportunidade de realizar este trabalho.

\section{REFERÊNCIAS}

1 CHIEREGATI, A. C.; PITARD, F. F. Fundamentos teóricos da amostragem. In: Arthur Pinto Chaves. (Org.). Teoria e prática do tratamento de minérios: manuseio de sólidos granulados. 1ed. São Paulo: Signus, 2011, v. 5, p. 299-338.

2 Gy,P. Sampling for analytical purpose. Traduzido por A.G. Royle, John Wiley \& Sons, West Sussex, Inglaterra.

3 PITARD, F.F. 1993. Pierre Gy's sampling theory and sampling practice: heterogeneity, sampling correctness, and statistical process control. 2nd ed., CRC Press, Boca Raton, Florida. 